\title{
Developmental differences in drawing performance of the dominant and non-dominant hand in right handed boys and girls
}

Citation for published version (APA):

van Mier, H. (2006). Developmental differences in drawing performance of the dominant and nondominant hand in right handed boys and girls. Human Movement Science, 25, 657-677. https://doi.org/10.1016/j.humov.2006.06.004

Document status and date:

Published: 01/01/2006

DOI:

10.1016/j.humov.2006.06.004

Document Version:

Publisher's PDF, also known as Version of record

Document license:

Taverne

Please check the document version of this publication:

- A submitted manuscript is the version of the article upon submission and before peer-review. There can be important differences between the submitted version and the official published version of record.

People interested in the research are advised to contact the author for the final version of the publication, or visit the DOI to the publisher's website.

- The final author version and the galley proof are versions of the publication after peer review.

- The final published version features the final layout of the paper including the volume, issue and page numbers.

Link to publication

\footnotetext{
General rights rights.

- You may freely distribute the URL identifying the publication in the public portal. please follow below link for the End User Agreement:

www.umlib.nl/taverne-license

Take down policy

If you believe that this document breaches copyright please contact us at:

repository@maastrichtuniversity.nl

providing details and we will investigate your claim.
}

Copyright and moral rights for the publications made accessible in the public portal are retained by the authors and/or other copyright owners and it is a condition of accessing publications that users recognise and abide by the legal requirements associated with these

- Users may download and print one copy of any publication from the public portal for the purpose of private study or research.

- You may not further distribute the material or use it for any profit-making activity or commercial gain

If the publication is distributed under the terms of Article $25 \mathrm{fa}$ of the Dutch Copyright Act, indicated by the "Taverne" license above, 


\title{
Developmental differences in drawing performance of the dominant and non-dominant hand in right-handed boys and girls
}

\author{
Hanneke van Mier * \\ Faculty of Psychology, Neurocognition, Maastricht University, P.O. Box 616, \\ 6200 MD Maastricht, The Netherlands \\ Available online 2 October 2006
}

\begin{abstract}
This paper addresses the development of fine motor skills in the dominant and non-dominant hand. A total of 60 right-handed children, aged 4-12 years old, were divided in five groups of 12 children, with six girls and six boys in each group. The children were presented with drawing tasks that had to be performed with the dominant and non-dominant hand. Small or large targets had to be connected by lines making either a zigzag (discrete) or slalom (continuous) movement. For each task, effects of age group, gender, hand, and target size were examined for drawing time, percentage of stop time, drawing distance, velocity, and errors. Comparison of stop times in both tasks showed that the zigzag task was performed in a discrete way while the slalom task was performed more continuously, except in the youngest children, who performed both tasks in a discrete manner. With increasing age the children performed the tasks faster, more accurate and with shorter stops. No significant differences were found between boys and girls. While a shorter drawing distance and less errors were observed for the dominant hand in both tasks, drawing time and velocity were not significantly different between both hands. However, the percentage of stop time was higher for the dominant hand. Moving to smaller targets resulted in slower and less accurate performance. A significant interaction of age group and hand was found for errors in both tasks, and for stop time and velocity in the slalom task, suggesting differential maturational changes for both hands in discrete and continuous drawing tasks.

(C) 2006 Elsevier B.V. All rights reserved.
\end{abstract}

\footnotetext{
* Tel.: +31 43 3884010; fax: +31433884125.

E-mail address: h.vanmier@psychology.unimaas.nl
} 
PsycINFO: $2800 ; 2330$

Keywords: Gender; Manual; Asymmetry; Discrete; Continuous

\section{Introduction}

Asymmetric hand dominance with usually more proficient performance of the dominant hand has been established in many studies in both adults (e.g., Annett, 2004; Brouwer, Sale, \& Nordstrom, 2001; Elliott \& Chua, 1996; Hammond, Bolton, Plant, \& Manning, 1988) and children (e.g., Annett, 2004; Bryden \& Roy, 2005; Poole et al., 2005). This asymmetry is also found in tasks involving both hands, with the dominant hand handling or manipulating an object that is held or stabilized by the non-dominant hand (Guiard, 1987; Wiesendanger, Kazennikov, Perrig, \& Kaluzny, 1996). Hand laterality is greatest for tasks involving fine motor sequential movements, like writing and drawing (Hammond, 2002; Phillips, Gallucci, \& Bradshaw, 1999; Provins, 1997), with the dominant or preferred hand clearly outperforming the non-dominant or non-preferred hand. Even people who are considered as being mixed handed or ambidextrous usually have a preferred hand for writing and drawing (Annett, 2004). Additionally, there is evidence suggesting hemispheric specialization for handwriting in right-handers (Mack, Gonzalez Rothi, \& Heilman, 1993).

The fact that in right-handed individuals, performance of the dominant right hand exceeds that of the non-dominant left hand for most motor tasks might be related to anatomical and functional asymmetries in the primary motor cortex (M1) of humans. Research suggests that the area of hand representation is greater in the dominant left hemisphere than in the non-dominant right hemisphere (Amunts et al., 1996; Amunts, Jancke, Mohlberg, Steinmetz, \& Zilles, 2000; Foundas, Hong, Leonard, \& Heilman, 1998; Hammond, 2002; Kim et al., 1993). Furthermore, research has shown that the motor representation in M1 undergoes continuous reorganization as an effect of training and experience, even in the adult brain (Sanes \& Donoghue, 2000). Based on findings that the digits of the preferred hand were more independent than those of the non-preferred hand, Reilly and Hammond (2004) hypothesized that the cortical circuits controlling the dominant hand have a greater potential for reorganization related to use and practice, resulting in superior skilled performance of the dominant hand.

Laterality effects for the arms are shown to be present already at the first couple of months after birth, with the right arm being more developed than the left, based on synchrony between the elbow and shoulder joints (Piek, Gasson, Barrett, \& Case, 2002). Comparing the layer-specific developmental pattern of M1 with functional data, Amunts, SchmidtPassos, Schleicher, and Zilles (1997) found that the structural maturation of interhemispheric asymmetry in the layers of M1 correlated with the development of hand preference.

Developmental studies investigating manual asymmetry have mainly focussed on general motor tasks (Largo, Fischer, \& Rousson, 2003; Smith, 1983), peg moving tasks (Fagard \& Corroyer, 2003; Poole et al., 2005; Roy, Bryden, \& Cavill, 2003) or (finger) tapping tasks (Fagard, 1987; Garvey et al., 2003; Njiokiktjien et al., 1997). Superior performance by the dominant hand has been reported in these studies. An interesting question is to what extent this lateral asymmetry in children is observed in fine motor tasks like writing and drawing and if this asymmetry changes with increasing age. To date, studies addressing developmental differences between both hands in paper and pencil tasks have 
been scarce with studies by Blank, Miller, and Von Voss (2000) and Robertson (2001) being the few studies reported in the literature. Research has shown that motor systems mature throughout school age (Bender, Weisbrod, Bornfleth, Resch, \& Oelkers-Ax, 2005; Chiarenza, Papakostopoulos, Giordana, \& Guareschi-Cazzullo, 1983; Diamond, 2000). We therefore tested children up to 12 years. If asymmetries between the hands are related to the amount of fine motor activities performed by the dominant hand as in writing and drawing, one might expect less asymmetry in children who have been less involved in these activities. We therefore also tested children aged 4 and 5 who were in Kindergarten and had hardly received any writing instruction.

Previous studies have shown improvement in fine motor performance, like drawing, with increasing age (Blank, Miller, Von Voss, \& Von Kries, 1999; Blank et al., 2000; Broderick \& Laszlo, 1987, 1988; Otte \& Van Mier, 2006; Robertson, 2001; Van Mier, Hulstijn, \& Meulenbroek, 1994). This improvement has been ascribed to maturation of corticospinal pathways, due to myelinization of corticospinal axons (Muller \& Homberg, 1992). While the myelinization process can explain age related improvement it cannot explain hand related differences or developmental differences between distally and proximally generated movements (Blank et al., 1999, 2000). This suggests that additional processes might account for maturational changes between hands in fine motor execution, like anatomical and functional asymmetries in the primary motor cortex (Amunts et al., 1996, 1997, 2000; Foundas et al., 1998; Hammond, 2002; Kim et al., 1993) or maybe differences in the motor command for the dominant and non-dominant hand movements (Priori et al., 1999).

Our study was set up to examine developmental profiles and hand laterality of discrete and continuous drawing movements. We therefore included two different tasks in our study; one task in which children had to connect targets by drawing zigzag lines between the targets, and a task in which targets were 'connected' by slalom movements. While the zigzag task consists of sequential discrete movements with each unit displaying a clear starting and end point, the slalom task is a discontinuous task in which starting and end point are context dependent. A discrete task is considered to be less difficult than a continuous task (Schmidt \& Lee, 2005).

A further objective of the current study was to address effects of task difficulty. According to Fitts' law, moving to smaller targets increases task difficulty (Fitts, 1954), resulting in slower and/or less accurate performance. This effect has been established in previous research in children (e.g., Lambert \& Bard, 2005; McKay \& Weir, 2004; Smits-Engelsman, Wilson, Westenberg, \& Duysens, 2003). Having children move to targets with a diameter of either 1 or $0.5 \mathrm{~cm}$ assessed effects of task difficulty.

An additional issue that was examined in the current study was related to that of gender differences. Few developmental studies have examined gender differences in manual motor tasks. In a peg moving task Poole et al. (2005) found that girls performed faster than boys. Others found no differences between boys and girls (Blank et al., 1999, 2000; Broderick \& Laszlo, 1988; Smith, 1983; Weil \& Amundsons, 1994). Largo et al. (2001, 2003), using different motor tasks, showed that gender differences were dependent on the task used. However, when they observed gender differences, they were usually very small. Some studies suggest gender differences in limb movements, with girls being more proficient in manual skills, whereas boys perform better in terms of gross motor skills (Anastasi, 1981; Thomas \& French, 1985). Research by Piek et al. (2002) has shown that these differences must be, at least partially, innate, rather than solely based on environmental factors. They 
observed greater coordination between the joints of the two arms for girls as opposed to more synchronous coupling of the legs for boys in children as young as 6 weeks.

Developmental research regarding asymmetrical hand differences related to gender has shown inconsistent results. While some researchers have reported larger intermanual differences for females, suggesting that they are more lateralized (Annett \& Kilshaw, 1983; Carlier, Dumont, Beau, \& Michel, 1993; Nalcaci, Kalaycioglu, Cicek, \& Genc, 2001; Pedersen, Sigmundsson, Whiting, \& Ingvaldsen, 2003), others observed the opposite, with more symmetry for female participants (Amunts et al., 2000; Carlier et al., 1993). Additionally, results have shown similar symmetry effects for both genders (Gabbard, Hart, \& Kanipe, 1993).

The current study was designed and conducted to examine how performance of the two hands would fluctuate as a function of age group and gender in discrete and continuous drawing tasks. Therefore, 4-12-year old boys and girls were instructed to draw as quickly and accurately as possible zigzag and slalom lines to targets of different sizes. We expected older children to perform better than younger ones, and better performance for movements made with the dominant hand and to larger targets. Based on inconsistent results reported in the literature no assumptions were made regarding differences between boys and girls.

\section{Methods}

\subsection{Participants}

Sixty right-handed children ( 30 girls and 30 boys) between the age of 4 and 12 years participated in the experiment. All participants visited the same primary school and had a normal intelligence. The children were divided in five age groups, with 12 children in each group. The youngest group ( $\mathrm{Gr} 1$ ) included children of 4 and 5 years old; the second group ( $\mathrm{Gr} 2$ ) consisted of children between 6 and 7 years; children in group 3 ( $\mathrm{Gr} 3$ ) were 8-9 years old; group 4 ( $\mathrm{Gr} 4$ ) had children between 10 and 11.5 years, while group 5 (Gr 5) consisted of children aged between 11.5 and 12.5 years. Mean ages (years: months, SD in months) in each group were respectively: 4:11 (SD 6), 7:2 (SD 6), 8:7 (SD 5), 10:6 (SD 4), and 12:1(SD 4). Each group consisted of six boys and six girls. Only the data of children who were able to perform both tasks were used. In the youngest age group (4-5 years) five children had to be replaced because they were not able to perform the slalom task, in the sense that they were not able to slalom around the targets. After having repeated the instruction and demonstrated the task again they still would not be able to connect the targets sequentially, would miss targets, make complete loops around the targets, connect the targets in a zigzag way, or mainly connect targets on one side. These errors were observed when slaloming around the large targets, so they were not related to differences in target size.

Handedness was assessed using a Dutch version of the Annett Handedness Inventory (1967) revised by Briggs and Nebes (1975). We modified this inventory for use by children by replacing two items of the original inventory (i.e., 'striking a match' and 'threading a needle') with items more convenient for children ('holding a knife when buttering bread' and 'eating with a spoon'). Hand preference for each item was scored on a five-point scale ranging from 'always left' (score -2) to 'always right' (score +2). This way scores ranged from -24 (strongly left-handed) to +24 (strongly right-handed). A person with a score of +9 or above is identified by Briggs and Nebes (1975) as right-handed. The parents or caretakers of 
the children administered the inventory at home. They were instructed to have their children perform the specific movement items and score accordingly. All children had a score of +17 or higher with mean scores and SD of each group being: Gr 1: +20.8 (SD 1.9); Gr 2: +23.3 (SD 1.5); Gr 3: +22.8 (SD 1.6); Gr 4: +23.1 (SD 1.8), and Gr 5: +22.0 (SD 1.9).

None of the children had any known neurological or motor disorder or any developmental or learning problems. All children showed proficient hand use regarding pen movements. The study was approved by the local Ethics Committee and the study was carried out according to the ethical guidelines laid down in the Declaration of Helsinki. Informed consent was obtained from the children and their parents/caretaker.

Each child received a monetary reward, which was paid to the school as was agreed upon with parents and school.

\subsection{Apparatus and material}

The drawing tasks were printed on A4 paper in landscape orientation and were taped on an A4-sized digitizing graphics tablet (WACOM, Intuous 2), which was connected to a Fujitsu-Siemens laptop computer. The $X$ and $Y$ position of a digitizing wireless ink pen was sampled at a frequency of $200 \mathrm{~Hz}$ with a spatial accuracy of $0.05 \mathrm{~mm}$. The pen left an ink trace on the paper, visible to the children. The laptop was out of sight of the children, so they could not see their performance on the monitor, making the task similar to a typical drawing task. The drawing trajectories were recorded and analyzed using OASIS software (de Jong, Hulstijn, Kosterman, \& Smits-Engelsman, 1996).

\subsubsection{Zigzag task}

In this task seven targets (boxes) had to be connected by straight zigzag lines (see Fig. 1, top). Boxes were either $10 \times 10 \mathrm{~mm}$ (large) or $5 \times 5 \mathrm{~mm}$ (small) and had to be connected in a vertical movement plane (defined as start and end point being in the vertical plane) or horizontal plane (defined as start and end point being in the horizontal plane). The opposite distance between the back walls of the boxes (inter-target distance) was fixed at $60 \mathrm{~mm}$.

\subsubsection{Slalom task}

In this task, children had to draw a slalom line through seven targets either in a vertical or horizontal plane (see Fig. 1, bottom). Size of and distance between the boxes was the same as in the zigzag task.

Pictures and stories were added to both tasks to make them more interesting and attractive for the children, stressing speed and accuracy to the same extent. The hungry mouse, which is looking for food in the holes, has to run fast to avoid being caught by the cat, while the skier has to be fast to win the game, both stressing speed. Running through the walls of the hole would make the hole accessible by the cat, while hitting the poles in the slalom task would result in penalty points, both stressing accuracy.

\subsection{Procedure}

The tasks were administered in a separate quiet area in the school. The children were seated at a school table and chair to assure a comfortable drawing position. Before the experiment started they were asked to write their name or draw letters or a figure (for the youngest age group), to get familiarized with the set-up. Additionally, the experimenter 


\section{Zigzag task}

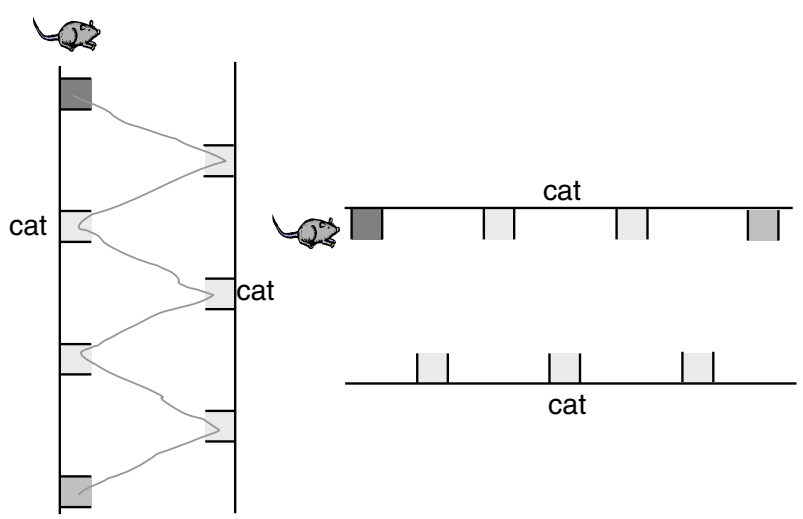

Slalom task

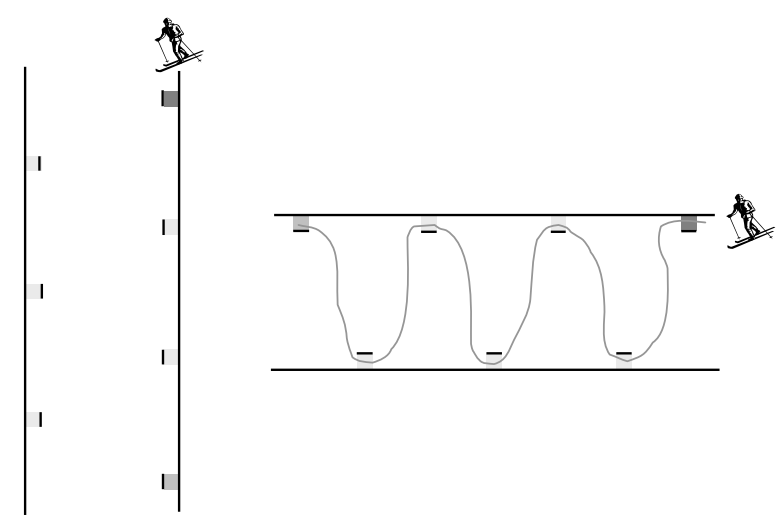

Fig. 1. Tasks used in the experiment. The zigzag task displays large targets for right hand performance (movements starting at the top at the left), the slalom task small targets for left hand performance (starting at the top at the right). Dark grey targets represent starting point, middle grey targets represent end point.

could confirm information regarding handedness obtained by the handedness questionnaires from the parents. Research has shown that writing/drawing hand correlates very highly with hand preference and performance (Corey, Hurley, \& Foundas, 2001).

The zigzag task was always administered first, as were the large targets. Both were considered less complex than the slalom task and the smaller targets. That way the task could be ended if performance on the zigzag task and/or large targets was unacceptable, avoiding unwanted frustration for the (younger) children. Presentation of drawing hand was counterbalanced between participants, of drawing plane also within participants. The tasks were administered once during two sessions of approximately half an hour on consecutive days, to prevent that children would miss too much of their classes that day. During one session the tasks were executed with the dominant hand and during the other session with the non-dominant hand. To control for biomechanical differences between the hands (Dounskaia, Van Gemmert, \& Stelmach, 2000; Phillips et al., 1999), movements made with the left hand were mirrored in relation to movements made with the right hand 
in both movement planes, so that homologous muscle groups were involved. Movements in the horizontal plane were either performed from left to right (performance with the dominant hand) or from right to left (performance with the non-dominant hand). Movements in the vertical plane were always performed in top-bottom direction. When the tasks were executed with the dominant hand, children always started at the left side of the trajectory, while drawing with the non-dominant hand always started at the right side (see Fig. 1).

Children were instructed to perform the tasks as quickly and accurately as possible with speed and accuracy being equally emphasized. They were also instructed to keep the pen on the paper once they had started the task. Before each task they were shown an example, and they received appropriate instructions. If needed, the children practiced the movements by tracing an example. Resting periods were included between each task.

\subsection{Data analysis}

Data records for all trials were low-pass filtered with a cutoff frequency of $10 \mathrm{~Hz}$ using a second order Butterworth filter and the drawing trajectories were displayed on the computer screen. The beginning and end of each trial were determined by the experimenter by means of the interactive computer program Oasis (de Jong et al., 1996). Because pen movements up to $2 \mathrm{~cm}$ above the tablet are also registered but are displayed in a different color than pen movements made on the tablet, the exact beginning and end of each trial could be easily and precisely determined and marked by the experimenter. For each task, movement duration, stop time, mean velocity and drawing distance were calculated for this period. Additionally, the number of errors was counted. Errors were always minor in the sense that they entailed only under- or overshooting a target or crossing a side wall (in the zigzag task) or the slalom pole (in the slalom task). As stated before, when a child made major errors, like missing target(s) or not connecting all targets sequentially, performance of that child was not included and another child was tested.

For each task a $2 \times 3$ repeated measures ANOVA was performed on each dependent variable, with age group (5) and gender (2) as between factors and hand (2), target size (2) and movement plane (2) as within factors. When a significant main effect was found for age group, comparisons between age groups were examined post-hoc using a Bonferroni correction.

\section{Results}

As said before, only when children were able to perform both tasks, their performance was included in the analyses. None of the children lifted the pen during task performance. Since only minor errors were observed, all trials were included in the analyses, resulting in a total of 16 (trials) $\times 60$ (children $)=960$ trials.

Because gender did not show significant main or interaction effects for any of the dependent variables, data were collapsed across gender. Movement plane did not show consistent effects. For some variables moving in the horizontal plane resulted in better performance, for others moving in the vertical plane. No significant interaction of movement plane and age group was found. We therefore also averaged over movement plane.

Target size showed the expected effect in the zigzag task for the percentage of stop time, drawing time, velocity and errors, with a significantly higher percentage of stop time 
$(p<.05)$, longer drawing times $(p<.01)$, higher velocity $(p<.001)$ and more errors $(p<.01)$ when moving to smaller targets. In the slalom task only a significant effect of size was found for distance and velocity (both $p<.001$ ), due to the fact that a longer path had to be taken to slalom around the larger targets resulting in a higher speed. No significant interaction of target size and age group was found except regarding the number of errors in the zigzag task $(p<.05)$. We therefore also averaged over target size (except for errors in the zigzag task). The above-mentioned averaging resulted for each task in a $1 \times 1$ repeated measures ANOVA, with age group (5) as the between factor and hand (2) as the within factor. Results of these analyses will be reported in the following sections.

Means and SD's of all variables averaged over gender, movement plane and target size, are given in Table 1 for all groups, for each task and for both hands.

Table 1

Means and SD (between brackets) of the percentage of stop time, drawing time, drawing distance, mean velocity and number of errors for each age group, task and hand

\begin{tabular}{|c|c|c|c|c|}
\hline \multirow[t]{2}{*}{ Group } & \multicolumn{2}{|l|}{ Zigzag task } & \multicolumn{2}{|l|}{ Slalom task } \\
\hline & Dom. hand & Non-dom. hand & Dom. hand & Non-dom. hand \\
\hline \multicolumn{5}{|c|}{ Percentage stop time } \\
\hline Gr 1 & $28.0(5.0)$ & $23.5(6.8)$ & $23.0(9.3)$ & $16.0(7.6)$ \\
\hline Gr 2 & $22.1(4.7)$ & $17.4(7.3)$ & $9.3(5.6)$ & $5.5(3.7)$ \\
\hline Gr 3 & $22.0(5.3)$ & $17.0(4.4)$ & $7.8(4.9)$ & $4.8(2.4)$ \\
\hline Gr 4 & $16.0(5.1)$ & $11.8(3.3)$ & $3.7(2.6)$ & $3.4(1.5)$ \\
\hline Gr 5 & $11.5(2.6)$ & $9.5(2.8)$ & $2.1(1.3)$ & $2.3(1.4)$ \\
\hline \multicolumn{5}{|c|}{ Drawing time $(s)$} \\
\hline Gr 1 & $10.4(3.8)$ & $11.1(2.6)$ & $14.0(4.3)$ & $13.3(3.2)$ \\
\hline Gr 2 & $8.5(3.0)$ & $8.9(3.1)$ & $11.0(3.2)$ & $11.2(2.9)$ \\
\hline Gr 3 & $7.4(1.8)$ & $7.9(1.6)$ & $10.1(1.9)$ & $10.1(1.7)$ \\
\hline Gr 4 & $7.2(1.5)$ & $7.7(1.2)$ & $8.7(1.8)$ & $9.7(1.7)$ \\
\hline Gr 5 & $6.1(1.5)$ & $7.1(1.7)$ & $7.5(1.4)$ & $8.8(1.3)$ \\
\hline \multicolumn{5}{|c|}{ Drawing distance $(\mathrm{cm})$} \\
\hline Gr 1 & $37.9(7.3)$ & $42.5(7.9)$ & $43.5(7.2)$ & $48.8(7.6)$ \\
\hline Gr 2 & $35.2(3.3)$ & $36.0(3.2)$ & $41.2(2.0)$ & $44.5(2.3)$ \\
\hline Gr 3 & $33.4(2.2)$ & $34.6(1.5)$ & $40.4(1.4)$ & $43.1(2.6)$ \\
\hline Gr 4 & $33.2(2.2)$ & $33.9(1.7)$ & $38.6(1.5)$ & 40.9 (1.9) \\
\hline Gr 5 & $31.8(1.4)$ & $33.1(1.5)$ & $38.7(1.9)$ & $41.3(2.4)$ \\
\hline \multicolumn{5}{|c|}{ Mean velocity $(\mathrm{cm} / \mathrm{s})$} \\
\hline Gr 1 & $2.60(0.6)$ & $2.89(0.5)$ & $2.56(7.2)$ & $3.05(0.6)$ \\
\hline Gr 2 & $3.42(1.4)$ & $3.53(1.3)$ & $3.56(2.0)$ & $3.75(0.9)$ \\
\hline Gr 3 & $3.53(0.9)$ & $3.59(0.7)$ & $3.69(1.4)$ & $4.11(0.9)$ \\
\hline Gr 4 & $3.88(1.1)$ & $3.61(0.9)$ & $4.33(1.5)$ & $4.15(1.0)$ \\
\hline Gr 5 & $4.82(1.4)$ & $4.33(1.2)$ & $5.20(1.9)$ & $4.62(0.7)$ \\
\hline \multicolumn{5}{|c|}{ Number of errors } \\
\hline Gr 1 & $2.69(1.2)$ & $3.60(0.8)$ & $3.00(2.6)$ & $4.21(2.4)$ \\
\hline Gr 2 & $0.52(0.7)$ & $1.85(1.2)$ & $0.23(0.3)$ & $0.50(0.7)$ \\
\hline Gr 3 & $0.31(0.4)$ & $1.15(1.0)$ & $0.19(0.2)$ & $0.46(0.4)$ \\
\hline Gr 4 & $0.19(0.4)$ & $0.63(0.8)$ & $0.08(0.2)$ & $0.40(0.3)$ \\
\hline Gr 5 & $0.40(0.6)$ & $0.60(0.8)$ & $0.06(0.2)$ & $0.29(0.4)$ \\
\hline
\end{tabular}

Dom. $=$ dominant, Gr $1=4-5$ years, Gr $2=6-7$ years, Gr $3=8-9$ years, Gr $4=10-11.5$ years, Gr $5=11.5-12$ years. 


\subsection{Discrete vs continuous movements}

To test if the children indeed performed the zigzag and slalom task differently, we first analyzed the percentage of movement time that was spent during stops in each task. The total stop time was divided by the total movement time and multiplied by 100 . An analysis including task as an additional within factor was performed. The results showed that during the zigzag task $17.9 \%$ of the total movement time was spent during stops, while only $7.7 \%$ of the time was stopped in the slalom task, a difference that was highly significant $(F(1,55)=393.99, p<.001)$. The effect of hand was also significant $(F(1,55)=31.33$, $p<.001)$, with a higher percentage of stop time for the dominant hand $(14.5 \%)$ vs the nondominant hand $(11.1 \%)$. There was also a highly significant interaction of task and age group $(F(4,55)=6.31, p<.001)$, caused by the fact that differences in the percentage of stop time between both tasks were small in the 4 and 5 your olds $(25.7 \%$ in the zigzag task and $19.5 \%$ in the slalom task), being much larger in the other age groups $(19.7 \%$ vs $7.4 \% ; 19.5 \%$ vs $6.3 \% ; 13.9 \%$ vs $3.5 \%$ and $10.5 \%$ vs $2.2 \%$ respectively, in groups $2-5$ ), as can be seen in Table 1 and Fig. 2. These data suggest that the youngest children performed both tasks in a similar way, using discrete movements in both tasks. Children age 6 and up displayed different movement strategies, with discrete movements and clear stops at the targets in the zigzag task and more continuous movements with considerably less stops in the slalom task. We therefore performed additional analyses for all dependent variables including only ages 6-12 (groups 2-5). Significance levels for all variables for main effects of age group and hand, as well as the interaction of age group by hand are given in Table 2, for the analyses including all age groups, and for the analyses including only groups $2-5$.

\subsection{Percentage of stop time}

\subsubsection{Zigzag task}

The analyses including all children showed a highly significant effect of age group $(F(4,55)=25.73, p<.001)$ and hand $(F(1,55)=30.97, p<.001)$ in the zigzag task. As shown in Table 1 and Fig. 2A, older children showed a smaller percentage of stop time. Contrary

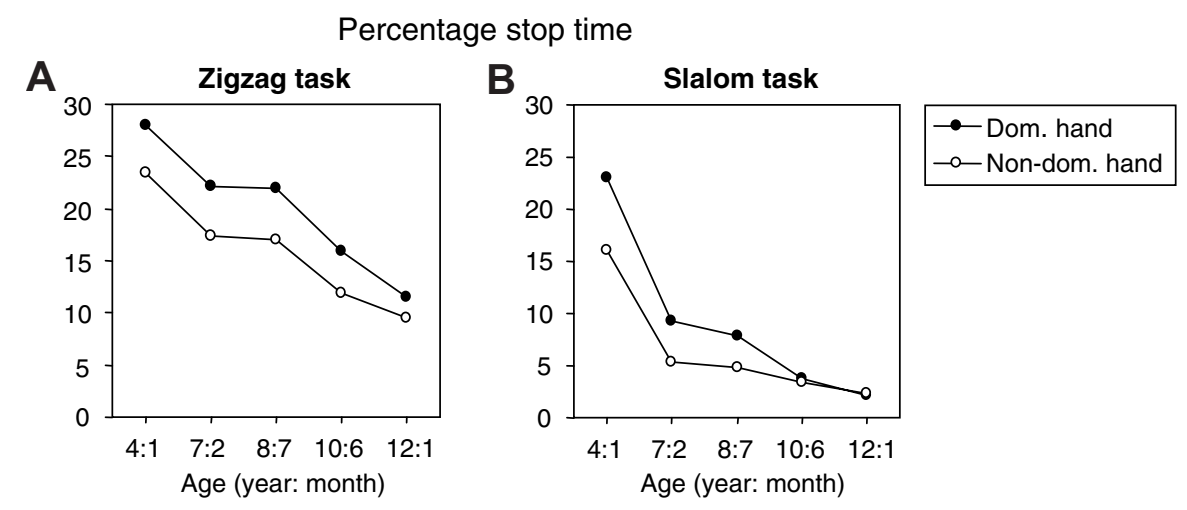

Fig. 2. Percentage of stop time for the dominant and non-dominant hand as an effect of age group for the zigzag (A) and slalom (B) task. Dom.= dominant. Note that the percentage of stop time was higher for the dominant hand. 
Table 2

Significance levels for each task for percentage of stop time, drawing time, drawing distance, velocity and errors, regarding the analyses including groups $1-5$ and groups $2-5$

\begin{tabular}{|c|c|c|c|c|c|c|c|c|c|c|}
\hline & \multicolumn{2}{|c|}{$\%$ Stop time } & \multicolumn{2}{|c|}{ Drawing time } & \multicolumn{2}{|c|}{ Drawing distance } & \multicolumn{2}{|c|}{ Velocity } & \multicolumn{2}{|l|}{ Errors } \\
\hline & Zigzag & Slalom & Zigzag & Slalom & Zigzag & Slalom & Zigzag & Slalom & Zigzag & Slalom \\
\hline \multicolumn{11}{|l|}{ Groups 1-5 } \\
\hline Age & $<.001$ & $<.001$ & $<.001$ & $<.001$ & $<.001$ & $<.001$ & $<.001$ & $<.001$ & $<.001$ & $<.001$ \\
\hline Hand & $<.001$ & $<.001$ & $<.08$ & ns & $<.01$ & $<.001$ & ns & ns & $<.001$ & $<.001$ \\
\hline Age $*$ hand & ns & $<.05$ & ns & ns & ns & ns & ns & $<.05$ & $<.01$ & $<.05$ \\
\hline \multicolumn{11}{|l|}{ Groups 2-5 } \\
\hline Age & $<.001$ & $<.001$ & $<.08$ & $<.01$ & $<.01$ & $<.001$ & $<.05$ & $<.01$ & $<.05$ & ns \\
\hline Hand & $<.001$ & $<.01$ & $<.01$ & $<.05$ & $<.05$ & $<.001$ & $\mathrm{~ns}$ & ns & $<.001$ & $<.001$ \\
\hline Age $*$ hand & ns & $<.05$ & ns & ns & ns & ns & ns & $<.05$ & $<.01$ & ns \\
\hline
\end{tabular}

to our expectations, the percentage of stop time was higher for the dominant hand $(19.9 \%)$ than for the non-dominant hand (15.8\%). This effect was the same in all age groups, given the fact that the interaction of hand and age group was not significant. Post-hoc comparisons between all age groups showed that the younger groups (1-3) had a higher percentage of stop time than the older groups (4 and 5). Group 1 stopped significantly longer than the other age groups $(p<.01$ compared to groups 2 and 3, $p<.001$ compared to groups 4 and 5). Group 2 stopped more than groups 4 and 5 ( $p<.01$ and $<.001$, respectively), and finally group 3 had a higher stop percentage that groups 4 and 5 ( $p<.05$ and $<.001$, respectively).

The analyses including only the children of 6-12 years old, showed the same results; only significant main effects for age group $(F(3,44)=17.18, p<.001)$ and hand $(F(1,44)=$ 26.53, $p<.001)$. Post-hoc comparisons between the ages showed that both groups 2 and 3 stopped significantly longer than group $4(p<.01)$ and $5(p<.001)$.

\subsubsection{Slalom task}

The analyses including all children showed also a highly significant effect of age group $(F(4,55)=35.01, p<.001)$ and hand $(F(1,55)=16.73, p<.001)$ in the slalom task. As can be seen in Table 1 and Fig. 2B, older children stopped less, with the percentage of stop time again being higher for the dominant hand $(9.2 \%)$ than for the non-dominant hand $(6.4 \%)$. There was also a significant interaction of hand and age group $(F(4,55)=3.61, p<.05)$, due to the fact that differences between both hands were largest in the younger age groups. Post-hoc comparisons between the age groups showed that group 1 had a higher percentage of stop time than all the older groups $(p<.001)$. Group 2 stopped significantly longer than group $5(p<.05)$.

The analyses including only the children of 6-12 years, showed the same pattern of results; a significant main effect for age group $(F(3,44)=10.10, p<.001)$ and hand $(F(1,44)=8.92, p<.01)$, as well as a significant interaction of hand and age group $(F(3,44)=2.87, p<.05)$. Again differences between both hands were more pronounced in the younger children. As said before, children aged 6 and older spent only a small percentage of the movement time while stopping (4.8\%), with more stops when performing with the dominant hand $(5.7 \%)$ than with the non-dominant hand (4.0\%). Post-hoc comparisons between the age groups showed that group 2 had a higher percentage of stop time than groups 4 and 5 ( $p<.01$ and $<.001$, respectively). Group 3 stopped significantly longer than group $5(p<.01)$. 


\subsection{Drawing time}

To calculate the drawing time, defined as the time that the pen was on the paper and moving, stop time was subtracted from the total movement time.

\subsubsection{Zigzag task}

The analyses including all children showed a highly significant effect of age group $(F(4,55)=7.66, p<.001)$ in the zigzag task. Older children had a shorter drawing time than younger ones, as presented in Table 1 and Fig. 3A. The effect of hand showed a trend $(F(1,55)=3.39, p<.08)$, with a mean drawing time of $7.93 \mathrm{~s}$ for the dominant hand and $8.51 \mathrm{~s}$ for the non-dominant hand. This effect was the same in all age groups. Post-hoc analyses between age groups showed that only group 1 had a significantly longer drawing time than groups 3 and $4(p<.01)$ and group $5(p<.001)$.

The analyses including only the children of 6-12 years old, showed reversed main effects; a trend for age group $(F(3,44)=2.44, p<.08)$ and a significant effect for hand $(F(1,44)=7.60, p<.01)$ with a mean drawing time of $7.32 \mathrm{~s}$ for the dominant hand and of $7.88 \mathrm{~s}$ for the non-dominant hand.

\subsubsection{Slalom task}

The analyses including all children showed a highly significant effect of age group $(F(4,55)=10.55, p<.001)$; as can be seen in Table 1 and Fig. $3 \mathrm{~B}$, also in the slalom task older children displayed shorter drawing times than younger children. Drawing times for the dominant (10.24s) and non-dominant hand (10.64 s) were not significantly different. Post-hoc analyses between age groups showed that group 1 had a significantly longer drawing time than group $3(p<.01)$ and groups 4 and $5(p<.001)$. Group 2 needed significantly more time than group $5(p<.05)$.

The analyses including only the children of 6-12 years showed a significant main effect for age group $(F(3,44)=5.36, p<.01)$ as well as for hand $(F(1,44)=6.20, p<.05)$, with drawing time for the dominant hand $(9.31 \mathrm{~s})$ being shorter than for the non-dominant hand (9.96 s). Post-hoc analyses between age groups showed that group 2 had a significantly longer drawing time than group $5(p<.01)$.

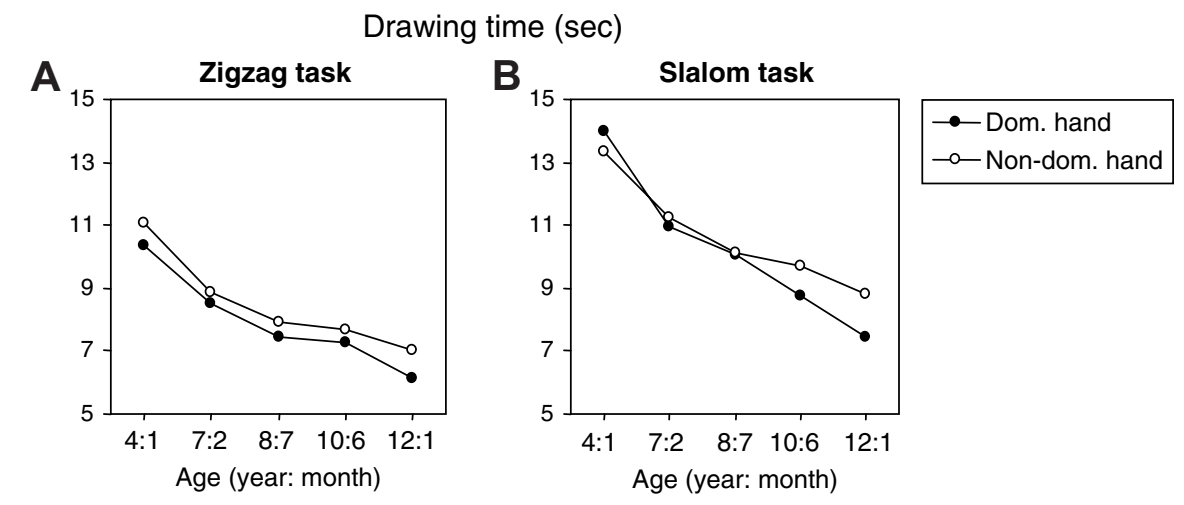

Fig. 3. Drawing time for the dominant and non-dominant hand as an effect of age group for the zigzag (A) and slalom (B) task. Dom. = dominant. Note that the drawing time is defined as the time that the pen was on the paper and moving. 


\subsection{Drawing distance}

\subsubsection{Zigzag task}

The analyses including all children showed a highly significant effect of age group $(F(4,55)=10.53, p<.001)$ in the zigzag task, with older children displaying a shorter drawing distance, as displayed in Table 1 and Fig. 4A. The effect of hand was also significant $(F(1,55)=8.80, p<.01)$, with a shorter drawing distance $(34.28 \mathrm{~cm})$ for the dominant hand than for the non-dominant hand $(36.01 \mathrm{~cm})$. This effect was found in all age groups. Posthoc analyses between age groups showed that drawing distance was significantly longer in group 1 compared to group $2(p<.01)$ and groups $3-5(p<.001)$.

The analyses including only the children of 6-12 years old, showed the same effects; a main effect for age group $(F(3,44)=6.57, p<.01)$ and hand $(F(1,44)=6.30, p<.05)$, with a mean distance of $33.39 \mathrm{~cm}$ for the dominant hand and of $34.30 \mathrm{~cm}$ for the non-dominant hand. Post-hoc comparisons between age groups showed that the significant effect of age group was caused by a significantly longer drawing distance of group 2 compared to groups 4 and 5 ( $p<.05$ and $<.001$, respectively).

\subsubsection{Slalom task}

The analyses including all children showed a highly significant effect of age group $(F(4,55)=8.32, p<.001)$; also in the slalom task older children displayed shorter drawing distances than younger children, as shown in Table 1 and Fig. 4B. Drawing distance for the dominant hand $(40.74 \mathrm{~cm})$ was significantly shorter than for the non-dominant hand $(43.44 \mathrm{~cm})(F(4,55)=25.66, p<.001)$. Post-hoc analyses between age groups showed that drawing distance was significantly longer in group 1 than in group $3(p<.01)$ and groups 4 and $5(p<.001)$.

The analyses including only the children of 6-12 years, showed a significant main effect for age group $(F(3,44)=7.39, p<.001)$ as well as for hand $(F(1,44)=115.78, p<.001)$. Mean distance for the dominant hand was $39.70 \mathrm{~cm}$, for the non-dominant hand $42.45 \mathrm{~cm}$. Post-hoc analyses between age groups showed that group 2 had a significantly longer drawing distance than groups 4 and $5(p<.01)$.

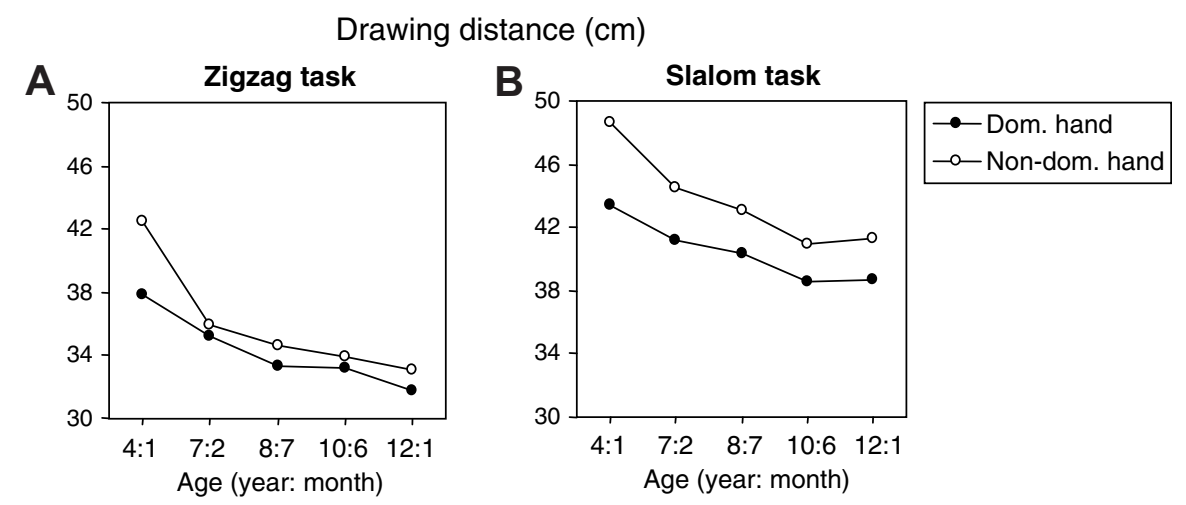

Fig. 4. Drawing distance for the dominant and non-dominant hand as an effect of age group for the zigzag (A) and slalom (B) task. Dom. = dominant. Note that the drawing distance in the slalom task was longer due to the fact that the line had to be drawn around the targets. 


\subsection{Mean velocity}

Mean velocity was calculated over the total movement time, including drawing time as well as stop time and is defined as the distance divided by the time in the selected segment.

\subsubsection{Zigzag task}

The analyses including all children showed a highly significant effect of age group $(F(4,55)=6.02, p<.001)$ in the zigzag task, with older children drawing faster than younger children, as displayed in Table 1 and Fig. 5A. Differences between both hands were not significant with almost similar velocities for the dominant hand $(3.7 \mathrm{~cm} / \mathrm{s})$ compared to the non-dominant hand $(3.6 \mathrm{~cm} / \mathrm{s})$. This effect was found in all age groups. Post-hoc analyses between age groups showed that groups 1 and 2 were slower than group $5(p<.001$ and $<.05$, respectively).

The analyses including only the children of 6-12 years old, showed the same effects; only a main effect for age group $(F(3,44)=2.97, p<.05)$. Post-hoc comparisons between age groups were not significant.

\subsubsection{Slalom task}

The analyses including all children showed a highly significant effect of age group $(F(4,55)=11.05, p<.001)$; also in the slalom task older children were faster than younger children, as shown in Table 1 and Fig. 5B. Again, the dominant and non-dominant hands performed at the same speed $(3.9 \mathrm{~cm} / \mathrm{s})$. There was a significant interaction of age group and hand $(F(4,55)=3.43, p<.05)$, due to the fact that children in the youngest 3 age groups performed faster with their non-dominant hand, while the older 2 groups were faster with their dominant hand, as can be seen in Fig. 5B. Post-hoc analyses between age groups showed that drawing speed was significantly slower in group 1 compared to group 3 , and group 3 compared to group 5 (both $p$ 's $<.05$ ), group 1 compared to group 4 , and group 2 compared to group 5 (both $p$ 's $<.01$ ), as well as in group 1 compared to group $5(p<.001)$.

The analyses including only the children of 6-12 years, showed a similar pattern as the analyses including all age groups; a significant main effect for age group $(F(3,44)=4.67$,

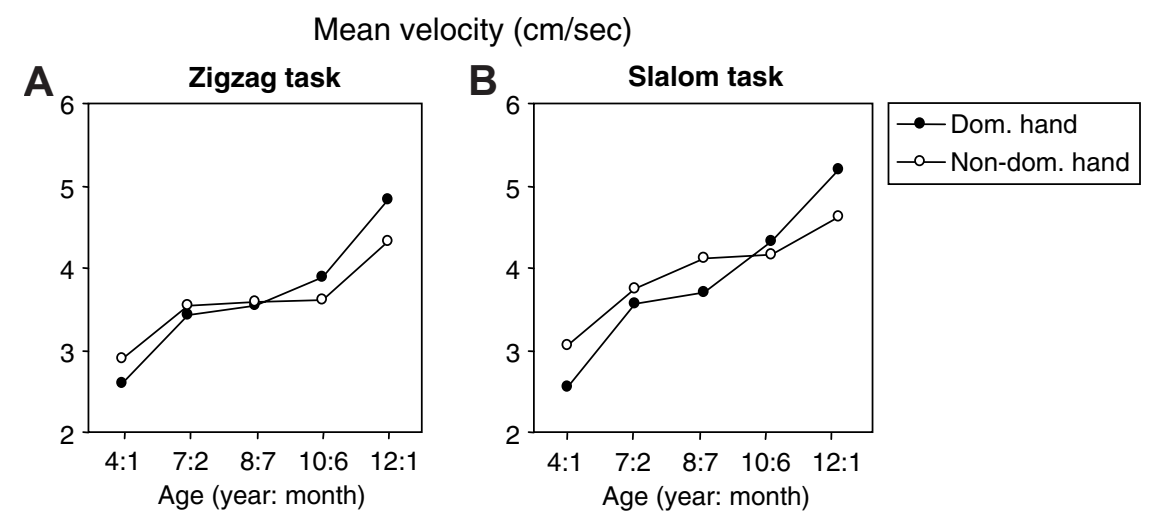

Fig. 5. Mean velocity for the dominant and non-dominant hand as an effect of age group for the zigzag (A) and slalom (B) task. Dom. = dominant. Note that the three younger groups were faster performing with their nondominant hand, while the two older groups showed faster performance with the dominant hand. 
$p<.01)$ and a significant interaction of age group and hand. Post-hoc analyses between age groups showed that groups 2 and 3 were significantly slower than group $5(p<.01$ and $<.05$, respectively).

\subsection{Number of errors}

\subsubsection{Zigzag task}

As stated before, because we found a significant interaction of target size and age group for number of errors in the zigzag task, we report here the results of the analysis including target size as a within factor. The analysis showed significant effects for age group $(F(4,55)=30.86, p<.001)$, hand $(F(1,55)=54.81, p<.001)$, and target size $(F(1,55)=13,46$, $p<.01)$. Table 1 and Fig. 6A summarize the effects of age group and hand, showing that older children made fewer errors than younger children and more accurate performance when drawing with the dominant hand (.82 errors) vs drawing with the non-dominant hand (1.57 errors). There was also a significant interaction of age group and hand $(F(4,55)=3.77, p<.01)$. Differences in errors between both hands were more pronounced in the younger age groups. The significant interaction of age group and target size $(F(4,55)=2.57, p<.05)$ was due to the fact that differences in errors between the target sizes were largest in the three youngest groups $(1.00, .54$ and .38 , respectively) and almost nonexistent in the two eldest groups (.06 and .04). Post-hoc analyses between age groups showed that only group 1 made significantly more errors than the other 4 groups $(p<.001)$.

The analyses including only the children of 6-12 years, showed only a significant interaction of age group and hand $(F(3,44)=5.33, p<.01)$ while the interaction of age group and target size was no longer significant $(p=.11)$. The main effects for age group and hand were both significant $(F(3,44)=3.48, p<.05$ for age group; $F(1,44)=43.37, p<.001$ for hand). Post-hoc analyses between age groups showed that only group 2 made significantly more errors than group $4(p<.05)$.

\subsubsection{Slalom task}

The analyses including all children showed a highly significant effect of age group $(F(4,55)=22.83, p<.001)$ and hand $(F(1,55)=16.25, p<.001)$. These effects are shown in

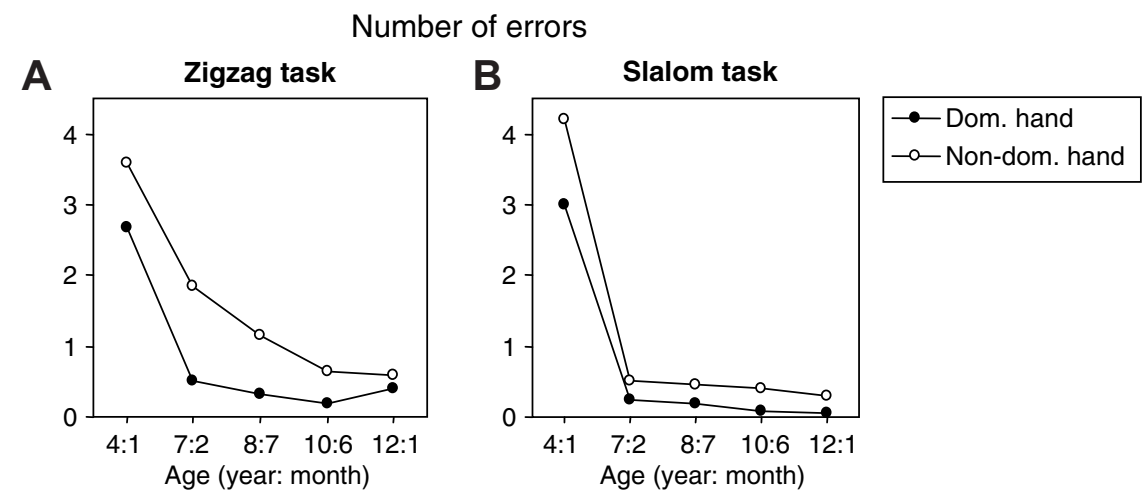

Fig. 6. Number of errors for the dominant hand and non-dominant hand as an effect of age group for the zigzag (A) and slalom (B) task. Dom.= dominant. Note that developmental changes were more pronounced for the non-dominant hand in the zigzag task. 
Table 1 and Fig. 6B displaying less errors for older children and for dominant hand drawing (.71) compared to drawing with the non-dominant hand (1.17). There was a significant interaction of age group and hand $(F(4,55)=2.73, p<.05)$ caused by the much larger difference in errors between both hands in the youngest age group. Post-hoc analyses between age groups showed that group 1 made significantly more errors than the other four groups $(p<.001)$.

The analyses including only the children of 6-12 years, showed a different pattern with only a significant effect for hand $(F(1,44)=20.49, p<.001)$. Differences between age groups were no longer significant $(p=.40)$.

\section{Discussion}

In this study developmental changes between the dominant and non-dominant hand were examined while boys and girls, aged 4-12 years, performed zigzag and slalom movements to targets of different size. Time spend during stops and actual drawing, drawing distance, velocity as well as accuracy of performance was measured. The results showed better performance with increasing age, while drawing with the dominant hand and when moving to larger targets.

Looking at the percentage of stop time in both tasks, the results showed that all children performed the zigzag task in a discrete manner, clearly stopping at each target. As for the slalom task, only children aged 6 years and up, performed this task more or less continuously. The youngest children of 4 and 5 years stopped almost as much in the slalom task as in the zigzag task, suggesting that they performed both tasks in a discrete manner.

One of the issues to be investigated in this study was the effect of age. As expected we found that older children stopped less, had a shorter drawing time and drawing distance, performed at a higher speed and made less errors, showing more accurate and more proficient drawing performance with increasing age. These findings are consistent with results reported in other developmental studies involving drawing tasks (Blank et al., 1999, 2000; Broderick \& Laszlo, 1987, 1988; Otte \& Van Mier, 2006; Robertson, 2001; Van Mier et al., 1994). Despite the fact that fixed distances were used between the targets, significant age related differences were observed for drawing distance, suggesting more proficient drawing performance with less correctional movements in older children. Although performance of the 4 and 5 year olds was especially inferior to performance of the older children, improvement continued throughout the age range investigated, as the analyses including only groups 2-5 showed (see Table 2). These age related effects were found in both tasks, showing developmental advancement in both discrete and continuous drawing movements for children age 6 and up. Only drawing time in the zigzag task and the number of errors made in the slalom task did not show age related effects in children of 6-12 years.

Age related decreases in the percentage of stop time, especially in the zigzag task, suggest more proficient planning and programming in older children. This might be due to more advance planning and programming of the sequence, in line with developmental results reported by Badan, Hauert, and Mounoud (2000) Yan, Thomas, Stelmach, and Thomas $(2000,2003)$ observed in sequential pointing and aiming tasks. Another option is that older children need less time to program and plan the upcoming segment during the stops due to more efficient information processing as has been suggested by other studies (Bourgeois \& Hay, 2003; Kail \& Salthouse, 1994; Miller \& Vernon, 1997; Van Mier et al., 1994). Even though the slalom task elicited less stop time, especially in older children, age 
related decreases were significant, suggesting age related efficiency of programming and planning in both tasks.

As for differences between the dominant and non-dominant hand, significant differences between the hands were found in both tasks for all variables except velocity and drawing time, when all age groups were included, and for all variables except velocity when groups 2-5 were analyzed. Zigzagging and slaloming with the dominant hand resulted in a shorter drawing distance, with differences between the hands being largest in the slalom task as can be seen in Fig. 4. This effect was seen in all age groups. Performance with the dominant hand resulted also in less errors in both tasks, being consistent with findings reported in developmental studies by Pellegrini, Andrade, and Teixeira (2004) in a reciprocal tapping task and Von Hofsten and Rösblad (1988) regarding absolute and systematic errors in manual pointing.

Our finding that all children performed at more or less the same speed with both hands confirms results reported by Hay and Velay (2003) in a pointing task. The children in our study complied with the instruction to perform the tasks as quickly as possible, even with the non-dominant hand. However, this resulted in significantly more errors when performing with the non-dominant hand, suggesting a speed-accuracy trade off (Fitts, 1954), also reported by others (Barral \& Debu, 2002; McKay \& Weir, 2004). Testing 5 year old boys and girls, Barral and Debu (2002) reported that girls were slower aiming with the nondominant hand compared to boys. However, spatial accuracy was lower in boys when aiming with the non-dominant hand, indicating a speed-accuracy trade off.

Contrary to our expectations, the percentage of stop time was smaller when performing with the non-dominant hand, an effect that was observed in all age groups being only similar for both hands in the eldest group in the slalom task. Shorter stops as well as a longer drawing distance observed for the non-dominant hand results in more proprioceptive information from the moving limb. Research by Sainburg and Kalakanis (2000) showed that hand trajectories and muscle and joint coordination patterns were systematically different between the hands in an arm reaching task. The results suggest that while the right hand might be highly proficient in accounting for muscle and inter joint interactions, the left hand might compensate by relying more on proprioceptive feedback. The role of proprioceptive information is most beneficiary in tasks involving online control of movement (Hay, Bard, Ferrel, Olivier, \& Fleury, 2005). A tendency to increase the size or better performance for larger letters or drawings has been reported in the literature when participants had to write or draw with the less skilled non-dominant hand (Blank et al., 2000; Robertson, 2001). In line with the former, longer drawing distances were observed for the non-dominant hand in our study, with asymmetries between the hands being largest in the slalom task. This longer drawing distance of the non-dominant hand in the slalom task might be due to more adjustments that had to be made to keep the number of errors in the slalom task to a minimum for the non-dominant hand.

Taking errors and drawing distance into account, in our study the dominant right hand clearly outperformed the non-dominant left hand, a finding that is often reported in the literature in writing and drawing tasks (Phillips et al., 1999) and is also found in developmental studies involving drawing (Blank et al., 2000; Robertson, 2001). Zigzagging with the non-dominant left hand resulted in more errors than with the dominant right hand, a result that confirms findings by Morgan et al. (1994) who studied differences between the hands in young (mean age 21 years) and older (mean age 70 years) adults in a zigzag task. In this task nine $10 \times 10 \mathrm{~mm}$ targets which were placed $12.5 \mathrm{~cm}$ apart had to be connected. 
Movements were made with both hands in the horizontal plane either moving from right to left or from left to right. They also reported significantly more errors for the non-dominant hand compared to the dominant hand.

A significant interaction between age group and hand was found with respect to speed and stop time in the slalom task and accuracy in the zigzag task (when including all age groups, this interaction was also found for accuracy in the slalom task). As for speed this interaction was caused by the fact that the three youngest age groups (ages 4-9) performed faster while slaloming with their non-dominant hand, while children aged 10-12 were faster with their dominant hand. Keep in mind that age related differences in the velocity data for both hands in the slalom task are related to differences in stop time, being shorter for the non-dominant hand in children aged 4-9 years, resulting in the significant interaction of age group and hand regarding the percentage of stop time. In the slalom task, errors between the hands were substantially larger in the youngest age group, while being more or less the same age 6 and up. This might indicate that children aged 6 and up are better able to use proprioceptive information to make online adjustments in the slalom task. Evidence for a rapid improvement of the proprioceptive system around this age has been shown in other studies (Hay et al., 2005; Von Hofsten \& Rösblad, 1988). The fact that the zigzag task has a higher end-point accuracy demand might account for the significant interaction between age group and hand, which was also found when only groups 2-5 were analyzed. A large decrease in errors was observed up to the age of 10 years. Performance with the less efficient non-dominant hand resulted in more errors, due to the crossing of inner and outer borders in all age groups.

In line with Fitts' law, target size showed a significant effect for all variables, with better performance when moving to the larger target size, a finding that has been reported in other developmental studies (Badan et al., 2000; Lambert \& Bard, 2005; McKay \& Weir, 2004; Pellegrini et al., 2004; Smits-Engelsman et al., 2003). Only with respect to errors a significant interaction between age group and target size was observed with differences in errors between both targets decreasing with age. Trying to perform as fast as possible resulted in more errors, especially in the youngest children, most likely due to higher end-point accuracy demands for the smaller targets. Based on these findings we cannot conclude that an increased target size was more beneficial for the younger children due to less efficient information processing in these children. As we did not measure reaction or initiation time we cannot rule out that the latter might have shown an effect on preparatory processes initiated before the drawing movements were started as was observed in a previous study (Van Mier et al., 1994).

None of our tasks or manipulations showed differences between girls and boys, consistent with findings in previous fine motor tasks (Blank et al., 1999, 2000; Broderick \& Laszlo, 1987, 1988; Weil \& Amundsons, 1994). Manual asymmetries between gender reported by others (Annett \& Kilshaw, 1983; Carlier et al., 1993; Carlier, Duyme, Capron, Dumont, \& Perez-Diaz, 1993; Nalcaci et al., 2001; Pedersen et al., 2003; Von Hofsten \& Rösblad, 1988) were not replicated in our study, rather, our findings are in line with results from Gabbard et al. (1993).

Based on research that has shown that right-handed participants, who use both hands professionally (like musicians or typists), display less manual asymmetry (Amunts et al., 1997; Jancke, Schlaug, \& Steinmetz, 1997; Provins \& Glencross, 1968), one might assume that asymmetry between the hands might be less pronounced in the future due to the fact that now-a-days even young children are using both hands more and more while operating 
a keyboard of a computer to 'write', instead of a pen or pencil. The fact that no pen or pencil was used to write, and most likely also review, this paper, illustrates this change in writing method.

The finding that children as young as 4 years were able to perform the tasks with the untrained non-dominant hand and the developmental changes observed for this hand despite effector-specific training of the dominant hand in right-handed participants, could be applied in the rehabilitation and training of the non-dominant hand in case of motor dysfunctions of the dominant hand.

\section{Conclusion}

In summary, distinct developmental profiles were found for both hands. The significant interaction of age group and hand regarding accuracy in the discrete zigzag task suggests that children up to the age of 10 years have problems performing fine motor movements with the non-dominant hand in tasks with high end-point accuracy demands. However, when proprioceptive information can be used to make adjustments in the movement path, as in the slalom task, children are more accurate with the non-dominant hand at a much younger age of 6 years. These data show differential maturational changes for both hands in discrete and continuous drawing tasks.

\section{Acknowledgements}

The author is grateful to the children, parents and teachers for their willingness to participate and collaborate in this study, and would like to thank Kim Huiskamp and Charlene Akse for recruiting and testing the children and analyzing part of the drawing data, and two anonymous reviewers for helpful comments.

\section{References}

Amunts, K., Jancke, L., Mohlberg, H., Steinmetz, H., \& Zilles, K. (2000). Interhemispheric asymmetry of the human motor cortex related to handedness and gender. Neuropsychologia, 38, 304-312.

Amunts, K., Schlaug, G., Schleicher, A., Steinmetz, H., Dabringhaus, A., Roland, P. E., et al. (1996). Asymmetry in the human motor cortex and handedness. NeuroImage, 4, 216-222.

Amunts, K., Schmidt-Passos, F., Schleicher, A., \& Zilles, K. (1997). Postnatal development of interhemispheric asymmetry in the cytoarchitecture of human area 4. Anatomy and Embryology, 196, 393-402.

Anastasi, A. (1981). Sex differences: Historical perspective and methodological implications. Developmental Review, 1, 187-206.

Annett, M. (1967). The binomial distribution of right, mixed and left handedness. Quarterly Journal of Experimental Psychology, 19, 327-333.

Annett, M. (2004). Hand preference observed in large healthy samples: Classification, norms and interpretations of increased non-right-handedness by the right shift theory. British Journal of Psychology, 95, 339-353.

Annett, M., \& Kilshaw, D. (1983). Right- and left-hand skill II: Estimating the parameters of the distribution of L-R differences in males and females. British Journal of Psychology, 74, 269-283.

Badan, M., Hauert, C. A., \& Mounoud, P. (2000). Sequential pointing in children and adults. Journal of Experimental Child Psychology, 75, 43-69.

Barral, J., \& Debu, B. (2002). Hand and gender differences in the organization of aiming in 5-year-old children. Neuropsychologia, 40, 152-161.

Bender, S., Weisbrod, M., Bornfleth, H., Resch, F., \& Oelkers-Ax, R. (2005). How do children prepare to react? Imaging maturation of motor preparation and stimulus anticipation by late contingent negative variation. NeuroImage, 27, 737-752. 
Blank, R., Miller, V., \& Von Voss, H. (2000). Human motor development and hand laterality: A kinematic analysis of drawing movements. Neuroscience Letters, 295, 89-92.

Blank, R., Miller, V., Von Voss, H., \& Von Kries, R. (1999). Effects of age on distally and proximally generated drawing movements: A kinematic analysis of school children and adults. Developmental Medicine and Child Neurology, 41, 592-596.

Bourgeois, F., \& Hay, L. (2003). Information processing and movement optimization during development: Kinematics of cyclical pointing in 5- to 11-year-old children. Journal of Motor Behavior, 35, 183-195.

Briggs, G. G., \& Nebes, R. D. (1975). Patterns of hand preference in a student population. Cortex, 11, 230-238.

Broderick, P., \& Laszlo, J. I. (1987). The drawing of squares and diamonds: A perceptual-motor task analysis. Journal of Experimental Child Psychology, 43, 44-61.

Broderick, P., \& Laszlo, J. I. (1988). The effects of varying planning demands on drawing components of squares and diamonds. Journal of Experimental Child Psychology, 45, 18-27.

Brouwer, B., Sale, M. V., \& Nordstrom, M. A. (2001). Asymmetry of motor cortex excitability during a simple motor task: Relationships with handedness and manual performance. Experimental Brain Research, 138, 467-476.

Bryden, P. J., \& Roy, E. A. (2005). Unimanual performance across the age span. Brain and Cognition, 57, $26-29$.

Carlier, M., Dumont, A. M., Beau, J., \& Michel, F. (1993). Hand performance of French children on a fingertapping test in relation to handedness, sex, and age. Perceptual and Motor Skills, 76, 931-940.

Carlier, M., Duyme, M., Capron, C., Dumont, A. M., \& Perez-Diaz, F. (1993). Is a dot-filling group test a good tool for assessing manual performance in children? Neuropsychologia, 31, 233-240.

Chiarenza, G. A., Papakostopoulos, D., Giordana, F., \& Guareschi-Cazzullo, A. (1983). Movement-related brain macropotentials during skilled performances. A developmental study. Electroencephalography and Clinical Neurophysiology, 56, 373-383.

Corey, D. M., Hurley, M. M., \& Foundas, A. L. (2001). Right and left handedness defined: A multivariate approach using hand preference and hand performance measures. Neuropsychiatry, Neuropsychology, and Behavioral Neurology, 14, 144-152.

de Jong, W. P., Hulstijn, W., Kosterman, B. J. M., \& Smits-Engelsman, B. C. M. (1996). OASIS software and its application in experimental handwriting research. In M. L. Simner, C. G. Leedham, \& A. J. W. M. Thomassen (Eds.), Handwriting and drawing research: Basic and applied issues (pp. 429-440). Amsterdam: IOS Press.

Diamond, A. (2000). Close interrelation of motor development and cognitive development and of the cerebellum and prefrontal cortex. Child and Development, 71, 44-56.

Dounskaia, N., Van Gemmert, A. W., \& Stelmach, G. E. (2000). Interjoint coordination during handwriting-like movements. Experimental Brain Research, 135, 127-140.

Elliott, D., \& Chua, R. (1996). Manual asymmetries in goal-directed movement. In D. Elliott \& E. A. Roy (Eds.), Manual asymmetries in motor performance (pp. 143-156). Boca Raton: CRC Press.

Fagard, J. (1987). Bimanual stereotypes: Bimanual coordination in children as a function of movements and relative velocity. Journal of Motor Behavior, 19, 355-366.

Fagard, J., \& Corroyer, D. (2003). Using a continuous index of laterality to determine how laterality is related to interhemispheric transfer and bimanual coordination in children. Developmental Psychobiology, 43, 44-56.

Fitts, P. M. (1954). The information capacity of the human motor system in controlling the amplitude of movement. Journal Experimental Psychology, 47, 381-391.

Foundas, A. L., Hong, K., Leonard, C. M., \& Heilman, K. M. (1998). Hand preference and magnetic resonance imaging asymmetries of the central sulcus. Neuropsychiatry, Neuropsychology, and Behavioral Neurology, 11, 65-71.

Gabbard, C., Hart, S., \& Kanipe, D. (1993). Hand preference consistency and fine motor performance in young children. Cortex, 29, 749-753.

Garvey, M. A., Ziemann, U., Bartko, J. J., Denckla, M. B., Barker, C. A., \& Wassermann, E. M. (2003). Cortical correlates of neuromotor development in healthy children. Clinical Neurophysiology, 114, 1662-1670.

Guiard, Y. (1987). Asymmetric division of labor in human skilled bimanual action: The kinematic chain as a model. Journal of Motor Behavior, 19, 486-517.

Hammond, G. (2002). Correlates of human handedness in primary motor cortex: A review and hypothesis. Neuroscience and Biobehavioral Reviews, 26, 285-292.

Hammond, G., Bolton, Y., Plant, Y., \& Manning, J. (1988). Hand asymmetries in interresponse intervals during rapid repetitive finger tapping. Journal of Motor Behavior, 20, 67-71.

Hay, L., Bard, C., Ferrel, C., Olivier, I., \& Fleury, M. (2005). Role of proprioceptive information in movement programming and control in 5 to 11-year old children. Human Movement Science, 24, 139-154. 
Hay, L., \& Velay, J-L. (2003). Interhemispheric relationships in 4- to 14-year-old children pointing to lateral targets. NeuroReport, 14, 1041-1044.

Jancke, L., Schlaug, G., \& Steinmetz, H. (1997). Hand skill asymmetry in professional musicians. Brain and Cognition, 34, 424-432.

Kail, R., \& Salthouse, T. A. (1994). Processing speed as a mental capacity. Acta Psychologica, 86, 199-225.

Kim, S. G., Ashe, J., Hendrich, K., Ellermann, J. M., Merkle, H., Ugurbil, K., et al. (1993). Functional magnetic resonance imaging of motor cortex: Hemispheric asymmetry and handedness. Science, 261, 615-617.

Lambert, J., \& Bard, C. (2005). Acquisition of visuomanual skills and improvement of information processing capacities in 6- to 10-year-old children performing a 2D pointing task. Neuroscience Letters, 377, 1-6.

Largo, R. H., Caflisch, J. A., Hug, F., Muggli, K., Molnar, A. A., Molinari, L., et al. (2001). Neuromotor development from 5 to 18 years. Part 1: Timed performance. Developmental Medicine and Child Neurology, 43, 436-443.

Largo, R. H., Fischer, J. E., \& Rousson, V. (2003). Neuromotor development from kindergarten age to adolescence: Developmental course and variability. Swiss Medical Weekly, 133, 193-199.

Mack, L., Gonzalez Rothi, L. J., \& Heilman, K. M. (1993). Hemispheric specialization for handwriting in right handers. Brain and Cognition, 21, 80-86.

McKay, S. M., \& Weir, P. L. (2004). The use of online control: A developmental perspective. Developmental Neuropsychology, 25, 299-320.

Miller, L. T., \& Vernon, P. A. (1997). Developmental changes in speed of information processing in young children. Developmental Psychology, 33, 549-554.

Morgan, M., Bradshaw, J. L., Phillips, J. G., Mattingley, J. B., Iansek, R., \& Bradshaw, J. A. (1994). Effects of hand and age upon abductive and adductive movements: A kinematic analysis. Brain and Cognition, 25, 194-206.

Muller, K., \& Homberg, V. (1992). Development of speed of repetitive movements in children is determined by structural changes in corticospinal efferents. Neuroscience Letters, 144, 57-60.

Nalcaci, E., Kalaycioglu, C., Cicek, M., \& Genc, Y. (2001). The relationship between handedness and fine motor performance. Cortex, 37, 493-500.

Njiokiktjien, C., de Sonneville, L., Hessels, M., Kurgansky, A., Vildavsky, V., \& Vranken, M. (1997). Unimanual and bimanual simultaneous fingertapping in school children: Developmental aspects and hand preferencerelated asymmetries. Laterality, 2, 117-135.

Otte, E., \& Van Mier, H. (2006). Bimanual interference in children performing a dual motor task. Human Movement Science, 25, 678-693.

Pedersen, A. V., Sigmundsson, H., Whiting, H. T. A., \& Ingvaldsen, R. P. (2003). Sex differences in lateralisation of fine manual skills in children. Experimental Brain Research, 149, 249-251.

Pellegrini, A. M., Andrade, E. C., \& Teixeira, L. A. (2004). Attending to the non-preferred hand improves bimanual coordination in children. Human Movement Science, 23, 447-460.

Phillips, J. G., Gallucci, R. M., \& Bradshaw, J. L. (1999). Functional asymmetries in the quality of handwriting movements: A kinematic analysis. Neuropsychology, 13, 291-297.

Piek, J. P., Gasson, N., Barrett, N., \& Case, I. (2002). Limb and gender differences in the development of coordination in early infancy. Human Movement Science, 21, 621-639.

Poole, J. L., Burtner, P. A., Torres, T. A., McMullen, C. K., Markham, A., Marcum, M. L., et al. (2005). Measuring dexterity in children using the nine-hole Peg Test. Journal of Hand Therapy, 18, 348-351.

Priori, A., Oliviero, A., Donati, E., Callea, L., Bertolasi, L., \& Rothwell, J. C. (1999). Human handedness and asymmetry of the motor cortical silent period. Experimental Brain Research, 128, 390-396.

Provins, K. A. (1997). The specificity of motor skill and manual asymmetry: A review of the evidence and its implications. Journal of Motor Behavior, 29, 183-192.

Provins, K. A., \& Glencross, D. J. (1968). Handwriting, typewriting and handedness. Quarterly Journal of Experimental Psychology, 20, 282-289.

Reilly, K. T., \& Hammond, G. R. (2004). Human handedness: Is there a difference in the independence of the digits on the preferred and non-preferred hands? Experimental Brain Research, 156, 255-262.

Robertson, S. D. (2001). Development of bimanual skill: The search for stable patterns of coordination. Journal of Motor Behavior, 33, 114-126.

Roy, E. A., Bryden, P., \& Cavill, S. (2003). Hand differences in pegboard performance through development. Brain and Cognition, 53, 315-317.

Sainburg, R. L., \& Kalakanis, D. (2000). Differences in control of limb dynamics during dominant and nondominant arm reaching. Journal of Neurophysiology, 83, 2661-2675.

Sanes, J. N., \& Donoghue, J. P. (2000). Plasticity and primary motor cortex. Annual Review of Neuroscience, 23, 393-415. 
Schmidt, R. A., \& Lee, T. D. (2005). Motor control and learning: A behavioral emphasis (4th ed.). Champaign, IL: Human Kinetics.

Smith, S. M. (1983). Performance difference between hands in children on the motor accuracy test-revised. American Journal of Occupational Therapy, 37, 96-101.

Smits-Engelsman, B. C., Wilson, P. H., Westenberg, Y., \& Duysens, J. (2003). Fine motor deficiencies in children with developmental coordination disorder and learning disabilities: An underlying open-loop control deficit. Human Movement Science, 22, 495-513.

Thomas, J. R., \& French, K. E. (1985). Gender differences across age in motor performance: A meta-analysis. Psychological Bulletin, 98, 260-282.

Van Mier, H., Hulstijn, W., \& Meulenbroek, R. G. J. (1994). Movement planning in children with motor disorders: Diagnostic implications of pattern complexity and previewing in copying. Developmental Neuropsychology, $10,231-254$.

Von Hofsten, C., \& Rösblad, B. (1988). The integration of sensory information in the development of precise manual pointing. Neuropsychologia, 26, 805-821.

Weil, M. J., \& Amundsons, S. J. (1994). Relationship between visuomotor and handwriting skills of children in kindergarten. American Journal of Occupational Therapy, 48, 982-988.

Wiesendanger, M., Kazennikov, O., Perrig, S., \& Kaluzny, P. (1996). Two hands - one action: The problem of bimanual coordination. In A. M. Wing, P. Haggard, \& J. R. Flanagan (Eds.), Hand and brain: The neurophysiology and psychology of hand movements (pp. 283-300). San Diego: Academic Press.

Yan, J. H., Thomas, J. R., Stelmach, G. E., \& Thomas, K. T. (2000). Developmental features of rapid aiming arm movements across the lifespan. Journal of Motor Behavior, 32, 121-140.

Yan, J. H., Thomas, K. T., Stelmach, G. E., \& Thomas, J. R. (2003). Developmental differences in children's ballistic aiming movements of the arm. Perceptual and Motor Skills, 96, 589-598. 